\title{
Linking of Bernstein-Chlodowsky and Szász-Appell-Kantorovich type operators
}

\author{
P. N. Agrawal ${ }^{\mathrm{a}}$, Dharmendra Kumar ${ }^{\mathrm{a}}$, Serkan Araci ${ }^{\mathrm{b}, *}$ \\ ${ }^{a}$ Department of Mathematics, Indian Institute of Technology Roorkee, Roorkee-247667, India. \\ ${ }^{b}$ Department of Economics, Faculty of Economics, Administrative and Social Sciences, Hasan Kalyoncu University, TR-27410, \\ Gaziantep, Turkey.
}

Communicated by M. Bohner

\begin{abstract}
In the present paper, we define a sequence of bivariate operators by linking the Bernstein-Chlodowsky operators and the Szász-Kantorovich operators based on Appell polynomials. First, we establish the moments of the operators and then determine the rate of convergence of these operators in terms of the total and partial modulus of continuity. Next, we obtain the order of approximation of the considered operators in a weighted space. Furthermore, we define the associated GBS (Generalized Boolean Sum) operators of the linking operators and then study the rate of convergence with the aid of the Lipschitz class of Bögel continuous functions and the mixed modulus of smoothness. (C)2017 All rights reserved.
\end{abstract}

Keywords: Appell polynomials, weighted approximation, GBS operators, partial and mixed modulus of smoothness, Peetre's K-functional.

2010 MSC: 41A25, 41A36, 41A63, 41A10.

\section{Introduction}

Verma and Tasdelen [17], linked the Szász operators and the orthogonal polynomials, e.g., Charlier polynomials, and introduced a sequence of positive linear operators to study the approximation of continuous functions of exponential growth. The generating function for the Charlier polynomials is given by

$$
e^{t}\left(1-\frac{t}{a}\right)^{u}=\sum_{k=0}^{\infty} C_{k}^{(a)}(u) \frac{t^{k}}{k !} .
$$

Agrawal and Ispir [1] introduced a bivariate operator associated with a combination of Bernstein-Chlodowsky and Szász-Charlier type operators and studied the degree of approximation of continuous functions. Jakimovski and Leviatan [13] proposed generalized Szász operators based on Appell polynomials as

$$
P_{n}(f ; x)=\frac{e^{-n x}}{g(1)} \sum_{k=0}^{\infty} p_{k}(n x) f\left(\frac{k}{n}\right),
$$

\footnotetext{
*Corresponding author

Email addresses: pnappfma@gmail.com (P. N. Agrawal), dharmendrak.dav@gmail .com (Dharmendra Kumar), mtsrkn@hotmail.com (Serkan Araci)

doi:10.22436/jnsa.010.06.39
} 
where $g(u) e^{u x}=\sum_{k=0}^{\infty} p_{k}(x) u^{k}$ is the generating function for the Appell polynomials $p_{k}(x) \geqslant 0$, with $g(z)=\sum_{n=0}^{\infty} a_{n} z^{n},|z|<R, R>1$, and $g(1) \neq 0$.

Atakut and Büyükyazici [2] introduced Stancu type modification of the operators (1.1) as

$$
P_{n}^{*}(f ; x)=\frac{e^{-b_{n} x}}{g(1)} \sum_{k=0}^{\infty} p_{k}\left(b_{n} x\right) f\left(\frac{k}{c_{n}}\right),
$$

where $\left(b_{n}\right),\left(c_{n}\right)$ denote the unbounded and increasing sequences of positive real numbers such that $b_{n} \geqslant 1, c_{n} \geqslant 1$, and $\lim _{n \rightarrow \infty} \frac{1}{c_{n}}=0, \frac{b_{n}}{c_{n}}=1+O\left(\frac{1}{c_{n}}\right)$, as $n \rightarrow \infty$ and proved some direct theorems.

In particular if $\mathrm{g}(z)=1$, then these operators reduce to the modified Szász operators studied by Walczak [18]. Further, if $b_{n}=n=c_{n}$, then we get the operators given by (1.1). Sidharth et al. [15] defined the Chlodowsky-Szász-Appell type operators and investigated the rate of convergence for a weighted space and the degree of approximation of the associated GBS operators. On the interval $\left[0, a_{n}\right]$ with $a_{n} \rightarrow \infty$, as $n \rightarrow \infty$, the Bernstein-Chlodowsky polynomials are given by

$$
B_{n}(f ; x)=\sum_{k=0}^{n}\left(\begin{array}{l}
n \\
k
\end{array}\right)\left(\frac{x}{a_{n}}\right)^{k}\left(1-\frac{x}{a_{n}}\right)^{n-k} f\left(k \frac{a_{n}}{n}\right),
$$

where $x \in\left[0, a_{n}\right]$ and $\lim _{n \rightarrow \infty} \frac{a_{n}}{n}=0$.

By combining the operators (1.2) and the Bernstein-Chlodowsky operators (1.3), we introduce the bivariate Kantorovich type operators as

$$
\begin{aligned}
L_{n, m}(f ; x, y)= & \frac{n}{a_{n}} c_{m} \sum_{k=0}^{n} \sum_{j=0}^{\infty}\left(\begin{array}{l}
n \\
k
\end{array}\right)\left(\frac{x}{a_{n}}\right)^{k}\left(1-\frac{x}{a_{n}}\right)^{n-k} \frac{e^{-b_{m} y}}{g(1)} p_{j}\left(b_{m} y\right) \\
& \times \int_{\frac{j}{c_{m}}}^{\frac{j+1}{c_{m}}} \int_{\frac{k}{n} a_{n}}^{\frac{k+1}{n} a_{n}} f(t ; s) d t d s
\end{aligned}
$$

for all $n, m \in \mathbb{N}, f \in C\left(A_{a_{n}}\right)$ with $A_{a_{n}}=\left\{(x, y): 0 \leqslant x \leqslant a_{n}, 0 \leqslant y<\infty\right\}$, and $C\left(A_{a_{n}}\right):=\{f$ : $A_{a_{n}} \rightarrow R$ is continuous $\}$. Note that the operator (1.4) is the tensorial product of ${ }_{x} B_{n}$ and $y P_{m}^{*}$, i.e., $T_{n, m}=$ ${ }_{x} \mathrm{~B}_{\mathrm{n}} \circ_{y} \mathrm{P}_{\mathrm{m}}^{*}$, where

$$
{ }_{x} B_{n}(f ; x, y)=\sum_{k=0}^{n}\left(\begin{array}{l}
n \\
k
\end{array}\right)\left(\frac{x}{a_{n}}\right)^{k}\left(1-\frac{x}{a_{n}}\right)^{n-k} \int_{\frac{k}{n} a_{n}}^{\frac{k+1}{n} a_{n}} f(t ; y) d t
$$

and

$$
{ }_{y} P_{m}^{*}(f ; x, y)=\frac{e^{-b_{m} y}}{g(1)} \sum_{k=0}^{\infty} p_{k}\left(b_{m} y\right) \int_{\frac{j}{c_{m}}}^{\frac{j+1}{c_{m}}} f(x ; s) d s .
$$

The purpose of the present paper is to establish the degree of approximation for the bivariate Kantorovich type operators defined in (1.4) by means of the moduli of continuity and the Lipschitz class. The rate of convergence of these operators for a weighted space is studied with the aid of modulus of continuity introduced in [11]. Subsequently, the GBS case of these operators (1.4) is introduced and the approximation degree for the GBS operators is obtained by means of the mixed modulus of smoothness.

\section{Preliminaries}

To examine the approximation properties of the operators (1.4), we give some basic results using the test functions $e_{i, j}=t^{i} s^{j}(i, j=0,1,2)$ as follows: 
Lemma 2.1. For the operators (1.4), we have

(i) $\mathrm{L}_{\mathrm{n}, \mathrm{m}}\left(e_{0,0} ; x, y\right)=1$,

(ii) $L_{n, m}\left(e_{1,0} ; x, y\right)=x+\frac{a_{n}}{2 n}$,

(iii) $L_{n, m}\left(e_{0,1} ; x, y\right)=\frac{b_{m}}{c_{m}} y+\frac{1}{c_{m}} \frac{g^{\prime}(1)}{g(1)}+\frac{1}{2 c_{m}}$,

(iv) $L_{n, m}\left(e_{2,0} ; x, y\right)=x^{2}+\frac{x}{n}\left(a_{n}-x\right)+\frac{a_{n}^{2}}{3 n^{2}}$,

(v) $L_{n, m}\left(e_{0,2} ; x, y\right)=\frac{b_{m}^{2}}{c_{m}^{2}} y^{2}+\frac{b_{m}}{c_{m}^{2}}\left(2 \frac{g^{\prime}(1)}{g(1)}+1\right) y+\frac{1}{c_{m}^{2}}\left(\frac{g^{\prime \prime}(1)}{g(1)}+\frac{g^{\prime}(1)}{g(1)}\right)+\frac{1}{3 c_{m}^{2}}$,

(vi) $L_{n, m}\left(e_{3,0} ; x, y\right)=x^{3}+\frac{3 a_{n} x^{2}}{n}\left\{\frac{1}{2}+\left(1-\frac{x}{a_{n}}\right)\right\}+\frac{a_{n}^{2} x}{n^{2}}\left\{\left(1-\frac{x}{a_{n}}\right)\left(1-\frac{2 x}{a_{n}}\right)+\frac{3}{2}\left(1-\frac{x}{a_{n}}\right)+1\right\}+\frac{a_{n}^{3}}{4 n^{3}}$,

(vii) $\mathrm{L}_{n, m}\left(e_{0,3} ; x, y\right)=\frac{b_{m}^{3}}{c_{m}^{3}} y^{3}+\frac{b_{m}^{2}}{c_{m}^{3}}\left(3 \frac{g^{\prime}(1)}{g(1)}+\frac{9}{2}\right) y^{2}+\frac{b_{m}}{c_{m}^{3}}\left(3 \frac{g^{\prime \prime}(1)}{g(1)}+6 \frac{g^{\prime}(1)}{g(1)}+\frac{7}{2}\right) y+\frac{1}{c_{m}^{3}}\left(\frac{g^{\prime \prime \prime}(1)}{g(1)}+\frac{9}{2} \frac{g^{\prime \prime}(1)}{g(1)}\right.$ $\left.+\frac{7}{2} \frac{g^{\prime}(1)}{g(1)}+\frac{1}{4}\right)$

(viii) $L_{n, m}\left(e_{4,0} ; x, y\right)=x^{4}\left(\frac{11}{n^{2}}-\frac{9}{n}+1\right)+\frac{a_{n} x^{3}}{n^{2}}\left(\frac{12}{n}-18\right)+\frac{a_{n}^{2} x^{2}}{n}\left(\frac{7}{n}-\frac{7}{n^{2}}+3\right)+\frac{a_{n}^{3} x}{n^{3}}$,

(ix) $L_{n, m}\left(e_{0,4} ; x, y\right)=\frac{b_{m}^{4}}{c_{m}^{4}} y^{4}+\frac{b_{m}^{3}}{c_{m}^{4}} y^{3}\left(\frac{g^{\prime}(1)}{g(1)}+\frac{39}{5}\right)+\frac{b_{m}^{2}}{c_{m}^{4}} y^{2}\left(6 \frac{g^{\prime \prime}(1)}{g(1)}+\frac{117}{5} \frac{g^{\prime}(1)}{g(1)}+11\right)+\frac{b_{m}}{c_{m}^{4}} y\left(4 \frac{g^{\prime \prime \prime}(1)}{g(1)}+\frac{117}{5} \frac{g^{\prime \prime}(1)}{g(1)}\right.$ $\left.+\frac{29}{5} \frac{g^{\prime}(1)}{g(1)}+\frac{31}{5}\right)+\frac{1}{c_{m}^{4}}\left(\frac{g^{(4)}(1)}{g(1)}+\frac{39}{5} \frac{g^{\prime \prime \prime}(1)}{g(1)}+\frac{18}{5} \frac{g^{\prime \prime}(1)}{g(1)}+\frac{31}{5} \frac{g^{\prime}(1)}{g(1)}+\frac{1}{5}\right)$.

Proof. By making simple calculations, we can easily prove the above results. Hence the details are omitted.

As a consequence of Lemma 2.1, we obtain:

Lemma 2.2. For the operator (1.4), we have the following results:

(i) $L_{n, m}\left(\left(e_{1,0}-x\right)^{2} ; x, y\right)=\frac{x}{n}\left(a_{n}-x\right)+\frac{a_{n}^{2}}{3 n^{2}}$,

(ii) $L_{n, m}\left(\left(e_{0,1}-y\right)^{2} ; x, y\right)=\left(\frac{b_{m}}{c_{m}}-1\right)^{2} y^{2}+\left(2 \frac{b_{m}}{c_{m}^{2}} \frac{g^{\prime}(1)}{g(1)}-\frac{2}{c_{m}} \frac{g^{\prime}(1)}{g(1)}+\frac{b_{m}}{c_{m}^{2}}\right) y+\frac{1}{c_{m}^{2}}\left(\frac{g^{\prime}(1)}{g(1)}+\frac{g^{\prime \prime}(1)}{g(1)}\right)+\frac{1}{3 c_{m}^{2}}$,

(iii) $L_{n, m}\left(\left(e_{1,0}-x\right)^{4} ; x, y\right)=\left(\frac{3}{n^{2}}-\frac{6}{n^{3}}\right) x^{4}-\frac{6 a_{n}(n-2)}{n^{3}} x^{3}+a_{n}^{2}\left(\frac{3}{n^{2}}-\frac{7}{n^{3}}\right) x^{2}+\frac{a_{n}^{3}}{n^{3}} x$,

(iv) $L_{n, m}\left(\left(e_{0,1}-y\right)^{4} ; x, y\right)=\left(\frac{b_{m}}{c_{m}}-1\right)^{4} y^{4}+\left\{\frac{b_{m}^{3}}{c_{m}^{4}}\left(\frac{g^{\prime}(1)}{g(1)}+\frac{39}{5}\right)+6 \frac{b_{m}}{c_{m}^{2}}\left(2 \frac{g^{\prime}(1)}{g(1)}+1\right)-4 \frac{b_{m}^{2}}{c_{m}^{3}}\left(3 \frac{g^{\prime}(1)}{g(1)}+\frac{9}{2}\right)-\right.$ $\left.\frac{4}{c_{m}} \frac{g^{\prime}(1)}{g(1)}\right\} y^{3}+\left\{\frac{b_{m}^{2}}{c_{m}^{4}}\left(6 \frac{g^{\prime \prime}(1)}{g(1)}+\frac{117}{5} \frac{g^{\prime}(1)}{g(1)}+11\right)-4 \frac{b_{m}}{c_{m}^{3}}\left(3 \frac{g^{\prime \prime}(1)}{g(1)}+6 \frac{g^{\prime}(1)}{g(1)}+72\right)+\frac{6}{c_{m}^{2}}\left(\frac{g^{\prime \prime}(1)}{g(1)}+\frac{g^{\prime}(1)}{g(1)}\right)\right\} y^{2}+\left\{\frac{b_{m}}{c_{m}^{4}}\right.$ $\left.\times\left(4 \frac{g^{\prime \prime \prime}(1)}{g(1)}+\frac{117}{5} \frac{g^{\prime \prime}(1)}{g(1)}+\frac{29}{5} \frac{g^{\prime}(1)}{g(1)}+\frac{31}{5}\right)-\frac{4}{c_{m}^{3}}\left(3 \frac{g^{\prime \prime \prime}(1)}{g(1)}+6 \frac{g^{\prime \prime}(1)}{g(1)}+\frac{7}{2} \frac{g^{\prime}(1)}{g(1)}\right)\right\} y+\frac{1}{c_{m}^{4}}\left(\frac{g^{4}(1)}{g(1)}+\frac{39}{5} \frac{g^{\prime \prime \prime}(1)}{g(1)}\right.$ $\left.+\frac{18}{5} \frac{g^{\prime \prime}(1)}{g(1)}+\frac{31}{5} \frac{g^{\prime}(1)}{g(1)}+\frac{1}{5}\right)$.

Lemma 2.3. Taking into account the conditions on $\left(a_{n}\right),\left(b_{n}\right),\left(c_{n}\right)$, and using Lemmas 2.1 and 2.2 , we are led to

(i) $L_{n, m}\left(\left(e_{1,0}-x\right)^{2} ; x, y\right) \leqslant C_{1}\left(\frac{a_{n}}{n}\right)\left(x^{2}+x+1\right)$ as $n \rightarrow \infty$,

(ii) $L_{n, m}\left(\left(e_{0,1}-y\right)^{2} ; x, y\right) \leqslant \frac{\eta(g)}{c_{m}}\left(y^{2}+y+1\right)$ as $m \rightarrow \infty$,

(iii) $\mathrm{L}_{n, m}\left(\left(e_{1,0}-x\right)^{4} ; x, y\right) \leqslant C_{2}\left(\frac{a_{n}}{n}\right)\left(x^{4}+x^{3}+x^{2}+x\right)$ as $n \rightarrow \infty$,

(iv) $L_{n, m}\left(\left(e_{0,1}-y\right)^{4} ; x, y\right) \leqslant \frac{\mu(g)}{c_{m}}\left(y^{4}+y^{3}+y^{2}+y+1\right)$ as $m \rightarrow \infty$,

where $\eta(\mathrm{g})$ and $\mu(\mathrm{g})$ are certain constants depending on $\mathrm{g}$.

\section{Main results}

In this section, we establish the degree of approximation of the operators given by (1.4) in the space of continuous functions on compact set $I_{a b}:=[0, a] \times[0, b] \subset A_{a_{n}}$. For $f \in C\left(I_{a b}\right)$, equipped with the norm $\|f\|_{C\left(I_{a b}\right)}=\sup _{(x, y) \in I_{a b}}|f(x, y)|$ the complete modulus of continuity for the bivariate case is defined 
as follows:

$$
\omega(f ; \delta)=\sup \left\{|f(t, s)-f(x, y)|:(t, s),(x, y) \in I_{a b} \text { and } \sqrt{(t-x)^{2}+(s-y)^{2}} \leqslant \delta\right\} .
$$

The partial moduli of continuity with respect to $x$ and $y$ is given by

$$
\begin{aligned}
& \omega_{1}(f ; \delta)=\sup \left\{\left|f\left(x_{1}, y\right)-f\left(x_{2}, y\right)\right|: y \in[0, b] \text { and }\left|x_{1}-x_{2}\right| \leqslant \delta\right\}, \\
& \omega_{2}(f ; \delta)=\sup \left\{\left|f\left(x, y_{1}\right)-f\left(x, y_{2}\right)\right|: x \in[0, a] \text { and }\left|y_{1}-y_{2}\right| \leqslant \delta\right\} .
\end{aligned}
$$

Evidently, these partial moduli of continuity satisfy the properties of the usual modulus of continuity.

Theorem 3.1. For all $(x, y) \in \mathrm{I}_{\mathrm{ab}}$ and $\mathrm{f} \in \mathrm{C}\left(\mathrm{I}_{\mathrm{ab}}\right)$, we have the following inequality:

$$
\left|L_{n, m}(f ; x, y)-f(x, y)\right| \leqslant 2 w\left(f ; \delta_{n, m}\right),
$$

where $\delta_{n, m}=\left(C_{1}\left(\frac{a_{n}}{n}\right)\left(x^{2}+x+1\right)+\frac{\eta(g)}{c_{m}}(y+1)^{2}\right)^{1 / 2}$.

Proof. From the definition of complete modulus of continuity, we have

$$
\begin{aligned}
\left|L_{n, m}(f ; x, y)-f(x, y)\right| & \leqslant L_{n, m}(|f(t, s)-f(x, y)| ; x, y) \\
& \leqslant L_{n, m}\left(\omega\left(f ; \sqrt{(t-x)^{2}+(s-y)^{2}}\right) ; x, y\right) \\
& \leqslant \omega\left(f ; \delta_{n, m}\right)\left\{1+\frac{1}{\delta_{n, m}} L_{n, m}\left(\sqrt{(t-x)^{2}+(s-y)^{2}} ; x, y\right)\right\} .
\end{aligned}
$$

Applying Cauchy-Schwarz inequaltiy and Lemma 2.3, we have

$$
\begin{aligned}
\left|L_{n, m}(f ; x, y)-f(x, y)\right| & \leqslant \omega\left(f ; \delta_{n, m}\right)\left[1+\frac{1}{\delta_{n, m}}\left\{L_{n, m}\left(\left(e_{1,0}-x\right)^{2}+\left(e_{0,1}-y\right)^{2} ; x, y\right)\right\}^{1 / 2}\right] \\
& \leqslant \omega\left(f ; \delta_{n, m}\right)\left[1+\frac{1}{\delta_{n, m}}\left\{L_{n, m}\left(\left(e_{1,0}-x\right)^{2} ; x, y\right)+L_{n, m}\left(\left(e_{0,1}-y\right)^{2} ; x, y\right)\right\}^{1 / 2}\right] \\
& \leqslant \omega\left(f ; \delta_{n, m}\right)\left[1+\frac{1}{\delta_{n, m}}\left\{O\left(\frac{a_{n}}{n}\right)\left(x^{2}+x+1\right)+\frac{\eta(g)}{c_{m}}(y+1)^{2}\right\}^{1 / 2}\right],
\end{aligned}
$$

from which the desired result is immediate.

Theorem 3.2. For $\mathrm{f} \in \mathrm{C}\left(\mathrm{I}_{\mathrm{ab}}\right)$ and all $(\mathrm{x}, \mathrm{y}) \in \mathrm{I}_{\mathrm{ab}}$, the following result holds:

$$
\left|L_{n, m}(f ; x, y)-f(x, y)\right| \leqslant 2\left(\omega_{1}\left(f ; \delta_{n}\right)+\omega_{2}\left(f ; \delta_{m}\right)\right),
$$

where

$$
\delta_{n}^{2}=L_{n, m}\left(\left(e_{1,0}-x\right)^{2} ; x, y\right)=\frac{x}{n}\left(a_{n}-x\right)+\frac{a_{n}^{2}}{3 n^{2}}
$$

and

$$
\begin{aligned}
\delta_{m}^{2}=L_{n, m}\left(\left(e_{0,1}-y\right)^{2} ; x, y\right)= & \left(\frac{b_{m}}{c_{m}}-1\right)^{2} y^{2}+\left(2 \frac{b_{m}}{c_{m}^{2}} \frac{g^{\prime}(1)}{g(1)}-\frac{2}{c_{m}} \frac{g^{\prime}(1)}{g(1)}+\frac{b_{m}}{c_{m}^{2}}\right) y \\
& +\frac{1}{c_{m}^{2}}\left(\frac{g^{\prime}(1)}{g(1)}+\frac{g^{\prime \prime}(1)}{g(1)}\right)+\frac{1}{3 c_{m}^{2}}
\end{aligned}
$$


Proof. Using the definition of the partial moduli of continuity, Lemma 2.2, and the Cauchy-Schwarz inequality, we have

$$
\begin{aligned}
\left|L_{n, m}(f ; x, y)-f(x, y)\right| & \leqslant L_{n, m}(|f(t, s)-f(x, y)| ; x, y) \\
& \leqslant L_{n, m}(|f(t, s)-f(x, s)| ; x, y)+L_{n, m}(|f(x, s)-f(x, y)| ; x, y) \\
& \leqslant L_{n, m}\left(\omega_{1}(f ;|t-x|) ; x, y\right)+L_{n, m}\left(\omega_{2}(f ;|s-y|) ; x, y\right) \\
& \leqslant \omega_{1}\left(f ; \delta_{n}\right)\left[1+\frac{1}{\delta_{n}} L_{n, m}(|t-x| ; x, y)\right]+\omega_{2}\left(f ; \delta_{m}\right)\left[1+\frac{1}{\delta_{m}} L_{n, m}(|s-y| ; x, y)\right] \\
& \leqslant \omega_{1}\left(f ; \delta_{n}\right)\left[1+\frac{1}{\delta_{n}}\left(L_{n, m}\left(\left(e_{1,0}-x\right)^{2} ; x, y\right)\right)^{1 / 2}\right] \\
& +\omega_{2}\left(f ; \delta_{m}\right)\left[1+\frac{1}{\delta_{m}}\left(L_{n, m}\left(\left(e_{0,1}-y\right)^{2} ; x, y\right)\right)^{1 / 2}\right] .
\end{aligned}
$$

This proves the result.

Now, we establish the degree of approximation for the bivariate operators (1.4) with the aid of Lipschitz class. For $0<\gamma_{1} \leqslant 1$ and $0<\gamma_{2} \leqslant 1$ and $f \in C\left(I_{a b}\right)$ we define the Lipschitz class $\operatorname{Lip} M\left(\gamma_{1}, \gamma_{2}\right)$ for the bivariate case as follows:

$$
|f(t, s)-f(x, y)| \leqslant M|t-x|^{\gamma_{1}}|s-y|^{\gamma_{2}}
$$

Theorem 3.3. Let $\mathrm{f} \in \operatorname{Lip}_{M}\left(\gamma_{1}, \gamma_{2}\right)$. Then, we have

$$
\left|L_{n, m}(f ; x, y)-f(x, y)\right| \leqslant M \delta_{n}^{\gamma_{1}} \delta_{m}^{\gamma_{2}}
$$

where $\delta_{n}$ and $\delta_{m}$ are the same as in Theorem 3.2.

Proof. Since $f \in \operatorname{Lip}_{M}\left(\gamma_{1}, \gamma_{2}\right)$, we may write

$$
\begin{aligned}
\left|L_{n, m}(f ; x, y)-f(x, y)\right| & \leqslant L_{n, m}(|f(t, s)-f(x, y)| ; x, y) \\
& \leqslant L_{n, m}\left(M|t-x|^{\gamma_{1}}|s-y|^{\gamma_{2}} ; x, y\right) \\
& \leqslant M_{x} B_{n}\left(|t-x|^{\gamma_{1}} ; x, y\right) \text { y } P_{m}^{*}\left(|s-y|^{\gamma_{2}} ; x, y\right) .
\end{aligned}
$$

Applying the Hölder's inequality with $\left(\mathrm{p}_{1}, \mathrm{q}_{1}\right)=\left(\frac{2}{\gamma_{1}}, \frac{2}{2-\gamma_{1}}\right)$ and $\left(\mathrm{p}_{2}, \mathrm{q}_{2}\right)=\left(\frac{2}{\gamma_{2}}, \frac{2}{2-\gamma_{2}}\right)$, we have

$$
\begin{aligned}
\left|L_{n, m}(f ; x, y)-f(x, y)\right| \leqslant & M_{x} B_{n}\left(\left(e_{1,0}-x\right)^{2} ; x, y\right)^{\gamma_{1} / 2}{ }_{x} B_{n}\left(e_{0,0} ; x, y\right)^{\left(2-\gamma_{1}\right) / 2} \\
& \times{ }_{y} P_{m}^{*}\left(\left(e_{0,1}-y\right)^{2} ; x, y\right)^{\gamma_{2} / 2}{ }_{y} P_{m}^{*}\left(e_{0,0} ; x, y\right)^{\left(2-\gamma_{2}\right) / 2} \\
= & M \delta_{n}^{\gamma_{1}} \delta_{m}^{\gamma_{2}} .
\end{aligned}
$$

This proves the theorem.

In the next theorem, we obtain the degree of approximation for the functions in $\mathrm{C}^{1}\left(\mathrm{I}_{\mathrm{ab}}\right)$, the space of continuous functions in $I_{a b}$ whose first order partial derivatives are continuous in $I_{a b}$, by the operators defined in (1.4).

Theorem 3.4. Let $\mathrm{f} \in \mathrm{C}^{1}\left(\mathrm{I}_{\mathrm{ab}}\right)$. Then we have

$$
\left|L_{n, m}(f ; x, y)-f(x, y)\right| \leqslant\left\|f_{x}^{\prime}\right\| \delta_{n}+\left\|f_{y}^{\prime}\right\| \delta_{m},
$$

where $\delta_{\mathrm{n}}$ and $\delta_{\mathrm{m}}$ are defined as in Theorem 3.2. 
Proof. From the hypothesis we can write

$$
f(t, s)-f(x, y)=\int_{x}^{t} f_{w}^{\prime}(w ; s) d w+\int_{y}^{s} f_{u}^{\prime}(x ; u) d u .
$$

Applying $L_{n, m}(. ; x, y)$ on both sides, we get

$$
\left|L_{n, m}(f ; x, y)-f(x, y)\right| \leqslant L_{n, m}\left(\left|\int_{x}^{t}\right| f_{w}^{\prime}(w ; s)|d w| ; x, y\right)+L_{n, m}\left(\left|\int_{y}^{s}\right| f_{u}^{\prime}(x ; u)|d u| ; x, y\right) .
$$

Since

$$
\left|\int_{x}^{t}\right| f_{w}^{\prime}(w ; s)|d w| \leqslant\left\|f_{x}^{\prime}\right\||t-x| \text { and }\left|\int_{y}^{s}\right| f_{u}^{\prime}(x ; u)|d u| \leqslant\left\|f_{y}^{\prime}\right\||s-y|
$$

we have

$$
\left|L_{n, m}(f ; x, y)-f(x, y)\right| \leqslant\left\|f_{x}^{\prime}\right\| L_{n, m}(|t-x| ; x, y)+\left\|f_{y}^{\prime}\right\| L_{n, m}(|s-y| ; x, y) .
$$

Applying the Cauchy-Schwarz inequality and using Lemma 2.1, we obtain

$$
\begin{aligned}
\left|L_{n, m}(f ; x, y)-f(x, y)\right| \leqslant & \left\|f_{x}^{\prime}\right\|\left(L_{n, m}\left((t-x)^{2} ; x, y\right)\right)^{1 / 2}\left(L_{n, m}\left(e_{0,0} ; x, y\right)\right)^{1 / 2} \\
& +\left\|f_{y}^{\prime}\right\|\left(L_{n, m}\left((s-y)^{2} ; x, y\right)\right)^{1 / 2}\left(L_{n, m}\left(e_{0,0} ; x, y\right)\right)^{1 / 2} \\
\leqslant & \left\|f_{x}^{\prime}\right\| \delta_{n}+\left\|f_{y}^{\prime}\right\| \delta_{m} .
\end{aligned}
$$

This completes the proof.

Let $C^{2}\left(I_{a b}\right)=\left\{f \in C\left(I_{a b}\right): f^{(i, j)} \in C\left(I_{a b}\right), 1 \leqslant i, j \leqslant 2\right\}$, where $f^{(i, j)}$ is the $(i, j)^{\text {th }}$ order partial derivative with respect to $x, y$ of $f$, endowed with the norm

$$
\|f\|_{C^{(2)}\left(I_{a b}\right)}=\|f\|_{C\left(I_{a b}\right)}+\|f\|_{C\left(I_{a b}\right)}^{1}+\|f\|_{C\left(I_{a b}\right)}^{2},
$$

where

$$
\|f\|_{C\left(I_{a b}\right)}^{1}=\sup _{(x, y) \in I_{a b}}\left\{|f(x, y)|,\left|f^{(1,0)}(x, y)\right|,\left|f^{(0,1)}(x, y)\right|\right\}
$$

and

$$
\|f\|_{C\left(I_{a b}\right)}^{2}=\sup _{(x, y) \in I_{a b}}\left\{|f(x, y)|,\left|f^{(1,0)}(x, y)\right|,\left|f^{(0,1)}(x, y)\right|,\left|f^{(2,0)}(x, y)\right|,\left|f^{(1,1)}(x, y)\right|,\left|f^{(0,2)}(x, y)\right|\right\} .
$$

Now, we proceed to estimate order of approximation of the sequence $L_{n, m}(f)$ to the function $f \in C\left(I_{a b}\right)$ in terms of the Peetre's K-functional given by

$$
\kappa(f ; \delta)=\inf _{g \in C^{2}\left(I_{a b}\right)}\left\{\|f-g\|_{C\left(I_{a b}\right)}+\delta\|g\|_{C^{2}\left(I_{a b}\right)}, \delta>0\right\} .
$$

It is also known [12] that the inequality

$$
\kappa(f ; \delta) \leqslant M_{1}\left\{\bar{\omega}_{2}(f ; \sqrt{\delta})+\min \{1, \delta\}\|f\|_{C\left(I_{a b}\right)}\right\}
$$

holds for all $\delta>0$, where the constant $M_{1}$ is independent of $f, \delta$ and $\bar{\omega}_{2}(f ; \sqrt{\delta})$ is the second order complete modulus of continuity. 
Theorem 3.5. If $\mathrm{f} \in \mathrm{C}\left(\mathrm{I}_{\mathrm{ab}}\right)$, then we have

$$
\left|L_{n, m}(f ; x, y)-f(x, y)\right| \leqslant M\left\{\bar{\omega}_{2}\left(f ; \sqrt{F_{n, m}(x, y)}\right)+\min \left\{1, F_{n, m}(x, y)\right\}\|f\|_{C\left(I_{a b}\right)}\right\}+\omega(f ; \delta),
$$

where $\delta=\left(\sqrt{\left(\frac{2 n x+a_{n}}{2 n}-x\right)^{2}+\left(\frac{2 g(1) b_{m} y+2 g^{\prime}(1)+g(1)}{2 c_{m} g(1)}-y\right)^{2}}\right)$ and $F_{n, m}(x, y)=O\left(\frac{a_{n}}{n}\right)\left(x^{2}+x+1\right)+O\left(\frac{1}{c_{m}}\right)$ $\times\left(y^{2}+y+1\right)$.

Proof. We define the following auxiliary operator

$$
L_{n, m}^{*}(f ; x, y)=L_{n, m}(f ; x, y)-f\left(\frac{2 n x+a_{n}}{2 n}, \frac{2 g(1) b_{m} y+2 g^{\prime}(1)+g(1)}{2 c_{m} g(1)}\right)+f(x, y) .
$$

Then by Lemma 2.2, we get

$$
L_{n, m}^{*}\left(e_{1,0} ; x, y\right)=x, \quad L_{n, m}^{*}\left(e_{0,1} ; x, y\right)=y .
$$

Hence,

$$
\mathrm{L}_{\mathrm{n}, \mathrm{m}}^{*}((\mathrm{t}-\mathrm{x}) ; \mathrm{x}, \mathrm{y})=0, \quad \mathrm{~L}_{\mathrm{n}, \mathrm{m}}^{*}((\mathrm{~s}-\mathrm{y}) ; \mathrm{x}, \mathrm{y})=0 .
$$

Let $g \in C^{2}\left(I_{a b}\right)$ and $t, s \in I_{a b}$. Then by Taylor's formula

$$
\begin{aligned}
g(t, s)-g(x, y) & =g(t, y)-g(x, y)+g(t, s)-g(t, y) \\
& =\frac{\partial g(x, y)}{\partial x}(t-x)+\int_{x}^{t}(t-u) \frac{\partial^{2} g(u, y)}{\partial u^{2}} d u+\frac{\partial g(x, y)}{\partial x}(s-y)+\int_{y}^{s}(s-v) \frac{\partial^{2} g(x, v)}{\partial v^{2}} d v .
\end{aligned}
$$

Applying the operator $\mathrm{L}_{\mathrm{n}, \mathrm{m}}^{*}(. ; x, y)$ on both sides of the above equation, we obtain

$$
\begin{aligned}
L_{n, m}^{*}(g ; x, y)-g(x, y)= & L_{n, m}^{*}\left(\int_{x}^{t}(t-u) \frac{\partial^{2} g(u, y)}{\partial u^{2}} d u ; x, y\right)+L_{n, m}^{*}\left(\int_{y}^{s}(s-v) \frac{\partial^{2} g(x, v)}{\partial v^{2}} d v ; x, y\right) \\
= & L_{n, m}\left(\int_{x}^{t}(t-u) \frac{\partial^{2} g(u, y)}{\partial u^{2}} d u ; x, y\right)-\int_{x}^{\frac{2 n x+a_{n}}{2 n}}\left(\frac{2 n x+a_{n}}{2 n}-u\right) \frac{\partial^{2} g(u, y)}{\partial u^{2}} d u \\
& +L_{n, m}\left(\int_{y}^{s}(s-v) \frac{\partial^{2} g(x, v)}{\partial v^{2}} d v ; x, y\right) \\
& -\int_{y}^{\frac{2 g(1) b_{m} y+2 g^{\prime}(1)+g(1)}{2 c_{m} g(1)}}\left(\frac{2 g(1) b_{m} y+2 g^{\prime}(1)+g(1)}{2 c_{m} g(1)}-v\right) \frac{\partial^{2} g(x, v)}{\partial v^{2}} d v .
\end{aligned}
$$

Hence using Lemma 2.2 and taking into account conditions on sequences $\left(a_{n}\right),\left(b_{n}\right)$, and $\left(c_{n}\right)$, we have

$$
\begin{aligned}
& \left|L_{n, m}^{*}(g ; x, y)-g(x, y)\right| \\
& \leqslant L_{n, m}\left(\left|\int_{x}^{t}\right|(t-u)\left|\frac{\partial^{2} g(u, y)}{\partial u^{2}}\right| d u \mid ; x, y\right) \\
& \quad+\int_{x}^{\frac{2 n x+a_{n}}{2 n}\left|\left(\frac{2 n x+a_{n}}{2 n}-u \frac{2 n x+a_{n}}{2 n}-u\right)\right|\left|\frac{\partial^{2} g(u, y)}{\partial u^{2}}\right| d u} \\
& \quad+L_{n, m}\left(\left|\int_{y}^{s}\right|(s-v)|| \frac{\partial^{2} g(x, v)}{\partial v^{2}}|d v| ; x, y\right) \\
& \quad-\int_{y}^{\frac{2 g(1) b_{m} y+2 g^{\prime}(1)+g(1)}{2 c_{m} g(1)}}\left|\left(\frac{2 g(1) b_{m} y+2 g^{\prime}(1)+g(1)}{2 c_{m} g(1)}-v\right)\right|\left|\frac{\partial^{2} g(x, v)}{\partial v^{2}}\right| d v
\end{aligned}
$$




$$
\begin{aligned}
\leqslant & \left\{L_{n, m}\left((t-x)^{2} ; x, y\right)+\left(\frac{2 n x+a_{n}}{2 n}-x\right)^{2}\right\}\|g\|_{C^{2}\left(I_{a b}\right)}+\left\{L_{n, m}\left((s-y)^{2} ; x, y\right)\right. \\
& \left.+\left(\frac{2 g(1) b_{m} y+2 g^{\prime}(1)+g(1)}{2 c_{m} g(1)}-y\right)^{2}\right\}\|g\|_{C^{2}\left(I_{a b}\right)} \\
\leqslant & \left(O\left(\frac{a_{n}}{n}\right)+\left(O\left(\frac{a_{n}}{n}\right)\right)^{2}+O\left(\frac{1}{c_{m}}\right)+\left(\frac{2 g(1)\left(b_{m}-c_{m}\right) y+2 g^{\prime}(1)+g(1)}{2 c_{m} g(1)}\right)^{2}\right)\|g\|_{C^{2}\left(I_{a b}\right)} \\
\leqslant & \left(O\left(\frac{a_{n}}{n}\right)+O\left(\frac{1}{c_{m}}\right)\right)\|g\|_{C^{2}\left(I_{a b}\right)} .
\end{aligned}
$$

Also, from (3.2) and Lemma 2.3,

$$
\left|L_{n, m}^{*}(f ; x, y)\right| \leqslant\left|L_{n, m}(f ; x, y)\right|+\left|f\left(\frac{2 n x+a_{n}}{2 n}, \frac{2 g(1) b_{m} y+2 g^{\prime}(1)+g(1)}{2 c_{m} g(1)}\right)\right|+|f(x, y)| \leqslant 3\|f\|_{C\left(I_{a b}\right)} .
$$

Therefore, for $f \in C\left(I_{a b}\right)$ and any $g \in C^{2}\left(I_{a b}\right)$

$$
\begin{aligned}
\left|L_{n, m}(f ; x, y)-f(x, y)\right| \leqslant & \left|L_{n, m}^{*}(f ; x, y)-f(x, y)+f\left(\frac{2 n x+a_{n}}{2 n}, \frac{2 g(1) b_{m} y+2 g^{\prime}(1)+g(1)}{2 c_{m} g(1)}\right)-f(x, y)\right| \\
\leqslant & \left|L_{n, m}^{*}((f-g) ; x, y)\right|+\left|L_{n, m}^{*}(g ; x, y)-g(x, y)\right|+|g(x, y)-f(x, y)| \\
& +\left|f\left(\frac{2 n x+a_{n}}{2 n}, \frac{2 g(1) b_{m} y+2 g^{\prime}(1)+g(1)}{2 c_{m} g(1)}\right)-f(x, y)\right| \\
\leqslant & 2\|f-g\|_{C\left(I_{a b}\right)}+\left(O\left(\frac{a_{n}}{n}\right)\left(x^{2}+x+1\right)+O\left(\frac{1}{c_{m}}\right)\left(y^{2}+y+1\right)\right)\|g\|_{C^{2}\left(I_{a b}\right)} \\
& +\left|f\left(\frac{2 n x+a_{n}}{2 n}, \frac{2 g(1) b_{m} y+2 g /(1)+g(1)}{2 c_{m} g(1)}\right)-f(x, y)\right| \\
\leqslant & 2\|f-g\|_{C\left(I_{a b}\right)}+\left(O\left(\frac{a_{n}}{n}\right)\left(x^{2}+x+1\right)+O\left(\frac{1}{c_{m}}\right)\left(y^{2}+y+1\right)\right)\|g\|_{C^{2}\left(I_{a b}\right)} \\
& +\omega\left(f ; \sqrt{\left(\frac{2 n x+a_{n}}{2 n}-x\right)^{2}+\left(\frac{2 g(1) b_{m} y+2 g^{\prime}(1)+g(1)}{2 c_{m} g(1)}-y\right)^{2}}\right) .
\end{aligned}
$$

Taking the infimum on the right-hand side over all $g \in \mathrm{C}^{2}\left(\mathrm{I}_{\mathrm{ab}}\right)$ and using inequality (3.1), we obtain

$$
\begin{aligned}
\left|L_{n, m}(f ; x, y)-f(x, y)\right| \leqslant & 2 \kappa\left(f ; F_{n, m}(x, y)\right)+\omega\left(f ; \sqrt{\left(\frac{2 n x+a_{n}}{2 n}-x\right)^{2}+\left(\frac{2 b_{m} y+1}{2 c_{m}}-y\right)^{2}}\right) \\
\leqslant & M\left\{\bar{\omega}_{2}\left(f ; \sqrt{F_{n, m}(x, y)}\right)+\min \left\{1, F_{n, m}(x, y)\right\}\|f\|_{C^{2}\left(I_{a b}\right)}\right\} \\
& +\omega\left(f ; \sqrt{\left(\frac{2 n x+a_{n}}{2 n}-x\right)^{2}+\left(\frac{2 g(1) b_{m} y+2 g^{\prime}(1)+g(1)}{2 c_{m} g(1)}-y\right)^{2}}\right) .
\end{aligned}
$$

This completes the proof.

Now, we estimate the degree of approximation of the bivariate operators (1.4) in a weighted space. Let $B_{\rho}$ be the space of all functions $f$ defined on $\mathbb{R}_{+} \times \mathbb{R}_{+}, \mathbb{R}_{+}=[0, \infty)$ having the property $|f(x, y)| \leqslant$ $M_{f} \rho(x, y)$, where $M_{f}$ is a positive constant depending only on $f$ and $\rho(x, y)=1+x^{2}+y^{2}$ is a weight function. Let $C_{\rho}$ be the subspace of $B_{\rho}$ of all continuous functions with the norm $\|f\|_{\rho}=\sup _{x, y \in \mathbb{R}_{0}^{+}} \frac{|f(x, y)|}{\rho(x, y)}$ 
and let $C_{\rho}^{0}$ be the subspace of all functions $f \in C_{\rho}$ such that $\lim _{\sqrt{x^{2}+y^{2}}}<\infty \frac{|f(x, y)|}{\rho(x, y)}$ exists finitely. For all $f \in C_{\rho}^{0}$, the weighted modulus of continuity [11] is defined by

$$
\omega_{\rho}\left(f ; \delta_{1}, \delta_{2}\right)=\sup _{x, y \in \mathbb{R}_{+}\left|h_{1}\right| \leqslant \delta_{1},\left|h_{2}\right| \leqslant \delta_{2}} \frac{\left|f\left(x+h_{1}, y+h_{2}\right)-f(x, y)\right|}{\rho(x, y) \rho\left(h_{1}, h_{2}\right)} .
$$

Further details of the weighted modulus of continuity can be found in [11].

Lemma 3.6 $([9,10])$. For the sequence of positive linear operators $\left\{S_{n, m}\right\}_{n, m \geqslant 1}$ acting from $C_{\rho}$ to $B_{\rho}$, it is necessary and sufficient that inequality

$$
\left\|S_{n, m}(\rho ; x, y)\right\|_{\rho} \leqslant k
$$

is fulfilled with some positive constant $k$.

Theorem $3.7([9,10])$. If a sequence of positive linear operators $S_{n, m}$, acting from $C_{\rho}$ to $B_{\rho}$, satisfies the conditions

$$
\begin{aligned}
& \lim _{n, m \rightarrow \infty}\left\|S_{n, m}\left(e_{00} ; x, y\right)-1\right\|_{\rho}=0, \\
& \lim _{n, m \rightarrow \infty}\left\|S_{n, m}\left(e_{10} ; x, y\right)-x\right\|_{\rho}=0, \\
& \lim _{n, m \rightarrow \infty}\left\|S_{n, m}\left(e_{01} ; x, y\right)-y\right\|_{\rho}=0, \\
& \lim _{n, m \rightarrow \infty}\left\|S_{n, m}\left(\left(e_{20}+e_{02}\right) ; x, y\right)-\left(x^{2}+y^{2}\right)\right\|_{\rho}=0,
\end{aligned}
$$

then, for any function $\mathrm{f} \in \mathrm{C}_{\rho}^{0}$,

$$
\lim _{n, m \rightarrow \infty}\left\|S_{n, m} f-f\right\|_{\rho}=0,
$$

and there exists a function $\mathrm{f}^{*} \in \mathrm{C}_{\rho} \backslash \mathrm{C}_{\rho}^{0}$, for which

$$
\lim _{n, m \rightarrow \infty}\left\|S_{n, m} f^{*}-f^{*}\right\|_{\rho} \geqslant 1 .
$$

Theorem $3.8([9,10])$. Let $S_{n, m}$ be a sequence of linear operators acting from $C_{\rho}$ to $B_{\rho}$, and let $\rho_{1}(x, y) \geqslant 1$ be a continuous function for which

$$
\lim _{|v| \rightarrow \infty} \frac{\rho(v)}{\rho_{1}(v)}=0,(\text { where } v=(x, y)) .
$$

If $S_{n, m}$ satisfies the conditions of Theorem 3.7 , then

$$
\lim _{n, m \rightarrow \infty}\left\|L_{n, m} f-f\right\|_{\rho_{1}}=0
$$

for all $\mathrm{f} \in \mathrm{C}_{\rho}$. Now, we consider the following positive linear operators $\mathrm{S}_{\mathrm{n}, \mathrm{m}}$, defined by

$$
S_{n, m}(f ; x, y)= \begin{cases}L_{n, m}(f ; x, y), & \text { when }(x, y) \in I_{a_{n} d_{m} \prime} \\ f(x, y), & \text { when }(x, y) \in R_{+}^{2} \backslash I_{a_{n} d_{m}}\end{cases}
$$

where $I_{a_{n} d_{m}}=\left\{(x, y): 0 \leqslant x \leqslant a_{n}, 0 \leqslant y \leqslant d_{m}\right\},\left(d_{m}\right)$ be a sequence such that $\lim _{m \rightarrow \infty} d_{m}=\infty$.

Theorem 3.9. Let $\rho(x, y)=1+x^{2}+y^{2}$ be weighted function and $S_{n, m}(f ; x, y)$ be a sequence of linear positive operators defined by (3.7). Then, for all $\mathrm{f} \in \mathrm{C}_{\rho}^{0}$, we have

$$
\lim _{n, m \rightarrow \infty}\left\|S_{n, m} f-f\right\|_{\rho_{1}}=0,
$$

where $\rho_{1}(x, y)$ is a continuous function satisfying condition in (3.6). 
Proof. First, we show that $S_{n, m}$ is acting from $C_{\rho}$ to $B_{\rho}$. Using Lemma 2.1, we can write

$$
\begin{aligned}
\left\|S_{n, m}(\rho ; x, y)\right\|_{\rho} \leqslant & 1+\sup _{(x, y) \in I_{a_{n} d_{m}}}\left\{\frac{1}{\rho(x, y)}+\left(1-\frac{1}{n}\right) \frac{x^{2}}{\rho(x, y)}+\frac{a_{n}}{n} \frac{x}{\rho(x, y)}+\frac{a_{n}^{2}}{3 n^{2} \rho(x, y)}\right. \\
& \left.+\frac{b_{m}^{2}}{c_{m}^{2}} \frac{y^{2}}{\rho(x, y)}+\frac{b_{m}}{c_{m}^{2}}\left(2 \frac{g^{\prime}(1)}{g(1)}+1\right) \frac{y}{\rho(x, y)}+\frac{1}{c_{m}^{2} \rho(x, y)}\left(\frac{g^{\prime \prime}(1)}{g(1)}+\frac{g^{\prime}(1)}{g(1)}+\frac{1}{3}\right)\right\} \\
\leqslant & 1+\varphi_{n, m}+\psi_{n, m},
\end{aligned}
$$

where $\varphi_{\mathrm{n}, \mathrm{m}}=1+\left(1-\frac{1}{n}\right)+\frac{b_{\mathrm{m}}^{2}}{c_{m}^{2}}$, and $\psi_{\mathrm{n}, \mathrm{m}}=\frac{\mathrm{b}_{\mathrm{m}}}{\mathrm{c}_{\mathrm{m}}^{2}}\left(2 \frac{g^{\prime}(1)}{g(1)}+1\right)+\frac{a_{n}}{n}+\frac{a_{n}^{2}}{3 n^{2}}+\frac{1}{c_{m}^{2}}\left(\frac{g^{\prime \prime}(1)}{g(1)}+\frac{g^{\prime}(1)}{g(1)}+\frac{1}{3}\right)$. Since $\lim _{n, m \rightarrow \infty} \varphi_{n, m}=3$ and $\lim _{n, m \rightarrow \infty} \psi_{n, m}=0$, there exists a positive constant $k$, such that $\varphi_{n, m}+\psi_{n, m}<k$ for all natural numbers $\mathrm{n}$ and $\mathrm{m}$. Hence, we have

$$
\left\|S_{n, m}(\rho ; x, y)\right\|_{\rho} \leqslant 1+k
$$

From Lemma 3.6, we have $S_{n, m}: C_{\rho} \rightarrow B_{\rho}$. If we can show that conditions of Theorem 3.7 are satisfied, then the proof of Theorem 3.9 is completed. Using Lemma 2.1, we can obtain (3.3)-(3.5). Finally, using Lemma 2.1, we get

$$
\left\|S_{n, m}\left(e_{20}+e_{02} ; x, y\right)-\left(x^{2}+y^{2}\right)\right\|_{\rho} \leqslant \psi_{n, m}
$$

and since $\lim _{n, m \rightarrow \infty} \psi_{n, m}=0$, we obtain the desired result.

Theorem 3.10. Let $\left\{S_{n, m}\right\}$ be a sequence of linear positive operators defined by (3.7). Then, for each function $\mathrm{f} \in \mathrm{C}_{\rho}$, we have

$$
\lim _{n, m \rightarrow \infty}\left\|S_{n, m} f-f\right\|_{\rho}=0 .
$$

Proof. From (3.3)-(3.5), we have

$$
\lim _{n, m \rightarrow \infty}\left\|S_{n, m}^{a}\left(e_{i j} ; x, y\right)-e_{i j}\right\|_{\rho}=0, i, j \in\{0,1\},
$$

and

$$
\lim _{n, m \rightarrow \infty}\left\|S_{n, m}^{a}\left(e_{20}+e_{02} ; x, y\right)-\left(e_{20}+e_{02}\right)\right\|_{\rho}=0,
$$

and using Theorem 3.9, we obtain the desired result.

Theorem 3.11. If $\mathrm{f} \in \mathrm{C}_{\rho}^{0}$, then for sufficiently large $\mathrm{n}, \mathrm{m}$, the following inequality holds:

$$
\sup _{x, y \in \mathbb{R}_{+}^{2}} \frac{\left|L_{n, m}(f ; x, y)-f(x, y)\right|}{\rho(x, y)^{3}} \leqslant C \omega_{\rho}\left(f ; \delta_{n}, \delta_{m}\right),
$$

where $\delta_{n}=\left(\frac{a_{n}}{n}\right)^{1 / 2}, \delta_{m}=\left(\frac{\sigma(g)}{c_{m}}\right)^{1 / 2}, \sigma(g)=\max \{\eta(g), \mu(g)\}$, and $C$ is a constant depending on $n, m$.

Proof. From [11, pp.577], we may write

$$
|f(t, s)-f(x, y)| \leqslant 8\left(1+x^{2}+y^{2}\right) \omega_{\rho}\left(f ; \delta_{n}, \delta_{m}\right)\left(1+\frac{|t-x|}{\delta_{n}}\right)\left(1+\frac{|s-y|}{\delta_{m}}\right) \times\left(1+(t-x)^{2}\right)\left(1+(s-y)^{2}\right) .
$$

Thus,

$$
\left|L_{n, m}(f ; x, y)-f(x, y)\right| \leqslant 8 \frac{n}{a_{n}} c_{m}\left(1+x^{2}+y^{2}\right) \omega_{\rho}\left(f ; \delta_{n}, \delta_{m}\right)
$$




$$
\begin{aligned}
& \times \sum_{k=0}^{n} \sum_{j=0}^{\infty}\left(\begin{array}{l}
n \\
k
\end{array}\right)\left(\frac{x}{a_{n}}\right)^{k}\left(1-\frac{x}{a_{n}}\right)^{n-k} \frac{e^{-b_{m} y}}{g(1)} p_{j}\left(b_{m} y\right) \\
& \times \int_{\frac{j}{c_{m}}}^{\frac{j+1}{c_{m}}} \int_{\frac{k}{n} a_{n}}^{\frac{k+1}{n} a_{n}}\left(1+\frac{1}{\delta_{n}}|t-x|\right)\left(1+\frac{1}{\delta_{m}}|s-y|\right)\left(1+(t-x)^{2}\right)\left(1+(s-y)^{2}\right) d t d s .
\end{aligned}
$$

Applying the Cauchy-Schwarz inequality, we have

$$
\begin{aligned}
\left|L_{n, m}(f ; x, y)-f(x, y)\right| \leqslant & 8\left(1+x^{2}+y^{2}\right)\left[1+L_{n, m}\left(\left(e_{1,0}-x\right)^{2} ; x, y\right)+\frac{1}{\delta_{n}} \sqrt{L_{n, m}\left(\left(e_{1,0}-x\right)^{2} ; x, y\right)}\right] \\
& \times \frac{1}{\delta_{n}} \sqrt{L_{n, m}\left(\left(e_{1,0}-x\right)^{2} ; x, y\right) L_{n, m}\left(\left(e_{1,0}-x\right)^{4} ; x, y\right)} \\
& \times\left[1+L_{n, m}\left(\left(e_{0,1}-y\right)^{2} ; x, y\right)+\frac{1}{\delta_{m}} \sqrt{L_{n, m}\left(\left(e_{0,1}-y\right)^{2} ; x, y\right)}\right. \\
& \left.\times \frac{1}{\delta_{m}} \sqrt{L_{n, m}\left(\left(e_{0,1}-y\right)^{2} ; x, y\right) L_{n, m}\left(\left(e_{0,1}-y\right)^{4} ; x, y\right)}\right] \omega_{\rho}\left(f ; \delta_{n}, \delta_{m}\right) .
\end{aligned}
$$

Using Lemma 2.3, we have

$$
\begin{aligned}
\left|L_{n, m}(f ; x, y)-f(x, y)\right| \leqslant & 8\left(1+x^{2}+y^{2}\right)\left[1+O\left(\frac{a_{n}}{n}\right)\left(x^{2}+x+1\right)+\frac{1}{\delta_{n}} \sqrt{O\left(\frac{a_{n}}{n}\right)\left(x^{2}+x+1\right)}\right. \\
& \left.+\frac{1}{\delta_{n}} \sqrt{O\left(\frac{a_{n}}{n}\right)\left(x^{2}+x+1\right) . O\left(\frac{a_{n}}{n}\right)\left(x^{4}+x^{3}+x^{2}+x\right)}\right] \times \omega_{\rho}\left(f ; \delta_{n}, \delta_{m}\right) \\
& \times\left[1+\frac{\eta(g)}{c_{m}}(y+1)^{2}+\frac{1}{\delta_{m}} \sqrt{\frac{\eta(g)}{c_{m}}(y+1)^{2}}+\frac{1}{\delta_{m}} \sqrt{\frac{\eta(g)}{c_{m}}(y+1)^{2} \frac{\mu(g)}{c_{m}}(y+1)^{4}}\right] .
\end{aligned}
$$

Thus we get the required result.

\section{Construction of GBS operators of Chlodowsky-Szász-Appell type}

The continuity and the differentiability of a function in Bögel space were first examined by Bögel in [5] and [6]. After this, Dobrescu and Matei [8] used the definitions of B-continuity and B-differentiability to obtain the approximating properties of GBS of bivariate Bernstein polynomials. In [3], Badea et al. proved the "Test function theorem" for the functions defined in the Bögel space of continuous functions. In the same space the quantitative variant of a Korovkin-type theorem was given by Badea et al. in [4]. Recently, Sidharth et al. [15] constructed the GBS operators of Bernstein-Schurer-Kantorovich type and obtained the degree of approximation for these operators. They also obtained the degree of approximation of B-continuous and B-differentiable functions by the GBS operators of q-Bernstein-Schurer-Stancu type [16]. Agrawal and Ispir [1] established the degree of approximation for the bivariate Chlodowsky-SzaszCharlier type operators and the associated GBS operators.

First we give some basic definitions and notations:

A real-valued function $f$ on the rectangle $A=([a, b] \times[c, d])$ is called B-continuous if for every $(x, y) \in$ A there holds

$$
\lim _{(u, v) \rightarrow(x, y)} \Delta_{(u, v)} f(x, y)=0,
$$

where $\Delta_{(u, v)} f(x, y)=f(x, y)-f(x, v)-f(u, y)+f(u, v)$.

We denote by $C_{b}(A)$, the space of all B-continuous functions on $A$. $B(A), C(A)$ denote the space of all bounded functions and the space of all continuous (in the usual sense) functions on $A$ endowed with the sup-norm $\|\cdot\|_{\infty}$. It is known that $C(A) \subset C_{b}(A)([7$, page 52]). 
The mixed modulus of smoothness of $f \in C_{b}(A)$ is defined as

$$
\omega_{\text {mixed }}\left(f ; \delta_{1}, \delta_{2}\right):=\sup \left\{\left|\Delta_{\left(x+h_{1}, y+h_{2}\right)} f(x, y)\right|\right\},
$$

where the supremum is taken over all $(x, y) \in A,\left(h_{1}, h_{2}\right) \in \mathbb{R}_{0}^{+} \times \mathbb{R}_{0}^{+}$, such that $\left(x+h_{1}, y+h_{2}\right) \in A$, $0<\left|h_{1}\right| \leqslant \delta_{1}, 0<\left|h_{2}\right| \leqslant \delta_{2}$, and where $\Delta_{\mathfrak{u}, v} f(x, y)$ is as defined above. The mixed modulus of continuity involving upper bounds and the total modulus of continuity were introduced by Marchaud [14].

A real-valued function defined on A is called uniformly B-continuous function if and only if

$$
\lim _{\delta_{1}, \delta_{2} \rightarrow 0} \omega_{\text {mixed }}\left(f ; \delta_{1}, \delta_{2}\right)=0 \text {. }
$$

Furthermore, for all non-negative numbers $\lambda_{1}, \lambda_{2}$ there holds

$$
\omega_{\text {mixed }}\left(f ; \lambda_{1} \delta_{1}, \lambda_{2} \delta_{2}\right) \leqslant(1+] \lambda_{1}[)(1+] \lambda_{2}[) \omega_{\text {mixed }}\left(f ; \delta_{1}, \delta_{2}\right),
$$

where $] \lambda[$ denotes the largest integer which is smaller than $\lambda$.

A function $f: A \longrightarrow R$ is called Bögel differentiable if for every $(x, y) \in A$,

$$
\lim _{(u, v) \rightarrow(x, y)} \frac{\Delta_{(u, v)} f(x, y)}{(u-x)(v-y)}=D_{B} f(x, y)<\infty .
$$

Here, $D_{B}$ is called the B-derivative of $f$ and the space of all B-differentiable functions is denoted by $D_{b}(A)$.

In this section, we introduce the GBS case of the operators defined in (1.4). For every $f \in C_{b}(A)$, the GBS operator associated with the operator $T_{n, m}(f ; x, y)$ is defined as follows:

$$
\begin{aligned}
\mathrm{u}_{n, m}(f ; x, y)= & \frac{n}{a_{n}} c_{m} \sum_{k=0}^{n} \sum_{j=0}^{\infty}\left(\begin{array}{l}
n \\
k
\end{array}\right)\left(\frac{x}{a_{n}}\right)^{k}\left(1-\frac{x}{a_{n}}\right)^{n-k} \frac{e^{-b_{m} y}}{g(1)} p_{j}\left(b_{m} y\right) \\
& \times \int_{\frac{j}{c_{m}}}^{\frac{j+1}{c_{m}}} \int_{\frac{k}{n} a_{n}}^{\frac{k+1}{n} a_{n}}\{f(t ; s)+f(x, s)-f(t, s)\} d t d s .
\end{aligned}
$$

Let $I_{c d}:=[0, c] \times[0, d] \subset A_{a_{n}}$.

Theorem 4.1. For every $f \in C_{b}\left(I_{c d}\right)$ and for all $(x, y) \in I_{c d}$, we have the following inequality for the operators defined in (4.1),

$$
\left|u_{n, m}(f ; x, y)-f(x, y)\right| \leqslant 4 \omega_{\text {mixed }}\left(f ; \delta_{n}, \delta_{m}\right),
$$

where $\delta_{n}=\left(\frac{a_{n}}{n}\left(c^{2}+c\right)\right)^{1 / 2}, \delta_{m}:=\delta_{m}(g)=\left(\frac{\rho(g)}{c_{m}}\right)^{1 / 2}$, and $\rho(g)$ is a constant depending on $\mathrm{g}$.

Proof. Using the definition of $\omega_{\text {mixed }}\left(f ; \delta_{n}, \delta_{m}\right)$ and the elementary inequality, we have

$$
\omega_{\text {mixed }}\left(f ; \lambda_{1} \delta_{n}, \lambda_{2} \delta_{m}\right) \leqslant\left(1+\lambda_{1}\right)\left(1+\lambda_{2}\right) \omega_{\text {mixed }}\left(f ; \delta_{n}, \delta_{m}\right), \lambda_{1}, \lambda_{2}>0 .
$$

Therefore,

$$
\begin{aligned}
\left|\Delta_{(x, y)} f(t, s)\right| & \leqslant \omega_{\text {mixed }}(f ;|t-x|,|s-y|) \\
& \leqslant\left(1+\frac{|t-x|}{\delta_{n}}\right)\left(1+\frac{|s-y|}{\delta_{m}}\right) \omega_{\text {mixed }}\left(f ; \delta_{n}, \delta_{m}\right)
\end{aligned}
$$

for every $(x, y),(t, s) \in I_{c d}$ and for any $\delta_{n}, \delta_{m}>0$. Further, by the definition of $\Delta_{(u, v)} f(x, y)$, we get

$$
f(x, s)+f(t, y)-f(t, s)=f(x, y)-\Delta_{(u, v)} f(x, y) .
$$


Applying the operator defined in (1.4) on both sides of the above equality, we get

$$
u_{n, m}(f ; x, y)=f(x, y) L_{n, m}\left(e_{0,0} ; x, y\right)-L_{n, m}\left(\Delta_{(x, y)} f(t, s) ; x, y\right) .
$$

Since $L_{n, m}\left(e_{0,0} ; x, y\right)=1$, using (4.2) and applying the Cauchy-Schwarz inequality,

$$
\begin{aligned}
& \left|U_{n, m}(f ; x, y)-f(x, y)\right| \\
& \leqslant L_{n, m}\left(\left|\Delta_{(x, y)} f(t, s)\right| ; x, y\right) \\
& \leqslant\left(L_{n, m}\left(e_{0,0} ; x, y\right)+\delta_{n}^{-1} \sqrt{L_{n, m}\left(\left(e_{1,0}-x\right)^{2} ; x, y\right)}+\delta_{m}^{-1} \sqrt{L_{n, m}\left(\left(e_{0,1}-y\right)^{2} ; x, y\right)}\right. \\
& \left.\quad+\delta_{n}^{-1} \delta_{m}^{-1} \sqrt{L_{n, m}\left(\left(e_{1,0}-x\right)^{2} ; x, y\right)} \sqrt{L_{n, m}\left(\left(e_{0,1}-y\right)^{2} ; x, y\right)}\right) \omega_{\text {mixed }}\left(f ; \delta_{n}, \delta_{m}\right) .
\end{aligned}
$$

By Lemma 2.3, we have

$$
L_{n, m}\left(\left(e_{1,0}-x\right)^{2} ; x, y\right)=\frac{x\left(a_{n}-x\right)}{n}+\frac{a^{2} n}{3 n^{2}} \leqslant C_{1} \frac{a_{n}}{n}\left(c^{2}+c+1\right) .
$$

Similarly

$$
L_{n, m}\left(\left(e_{0,1}-y\right)^{2} ; x, y\right) \leqslant \frac{\eta(g)}{c_{m}}\left(y^{2}+y+1\right) \leqslant \frac{\eta(g)}{c_{m}}\left(d^{2}+d+1\right)=\frac{\rho(g)}{c_{m}} .
$$

Hence we get the required result.

In our next theorem, we obtain the degree of approximation of the operators $u_{n, m}$ by means of the Lipschitz-class which is defined as follows:

If $f \in C_{b}\left(I_{c d}\right)$, for two parameters $0<\xi_{1} \leqslant 1$ and $0<\xi_{2} \leqslant 1, \operatorname{Lip}_{M}\left(\xi_{1}, \xi_{2}\right)$ is defined by

$$
\operatorname{Lip}_{M}\left(\xi_{1}, \xi_{2}\right)=\left\{f \in C_{b}\left(I_{c d}\right):\left|\Delta_{(x, y)} f(t, s)\right| \leqslant M|t-x|^{\xi_{1}}|s-y|^{\xi_{2}} \text { for }(t, s),(x, y) \in I_{c d}\right\} .
$$

Theorem 4.2. For $f \in \operatorname{Lip}_{M}\left(\xi_{1}, \xi_{2}\right)$ and $(x, y) \in I_{c d}$, we have

$$
\left|u_{n, m}(f ; x, y)-f(x, y)\right| \leqslant M \delta_{n}^{\xi_{1} / 2} \delta_{m}^{\xi_{2} / 2},
$$

where $\delta_{n}=\left\|_{x} B_{n}\left((t-x)^{2} ; \cdot\right)\right\|_{\infty}, \delta_{m}=\left\|_{y} P_{m}^{*}\left((s-y)^{2} ; \cdot\right)\right\|_{\infty}$, and $M$ is a certain positive constant.

Proof. By the definition of $U_{n, m}(f ; \cdot, \cdot)$ and using the linearity of the operator $T_{n, m}(f ; \cdot, \cdot)$, we may write

$$
\begin{aligned}
\mathrm{U}_{n, m}(f ; \cdot, \cdot) & =\mathrm{L}_{n, m}(f(x, s)+f(t, y)-f(t, s) ; x, y) \\
& =L_{n, m}\left(f(x, y)-\Delta_{(t, s)} f(x, y) ; x, y\right) \\
& =f(x, y) L_{n, m}\left(e_{0,0} ; x, y\right)-L_{n, m}\left(\Delta_{(t, s)} f(x, y) ; x, y\right) .
\end{aligned}
$$

By our hypothesis, we get

$$
\begin{aligned}
\left|\mathrm{U}_{n, m}(f ; x, y)-f(x, y)\right| & \leqslant L_{n, m}\left(\left|\Delta_{(t, s)} f(x, y)\right| ; x, y\right) \\
& \leqslant M L_{n, m}\left(|t-x|^{\xi_{1}}|s-y|^{\xi_{2}} ; x, y\right) \\
& =M L_{n, m}\left(|t-x|^{\xi_{1}} ; x, y\right) L_{n, m}\left(|s-y|^{\xi_{2}} ; x, y\right) .
\end{aligned}
$$

Now, applying the Hölder's inequality with $\left(p_{1}, q_{1}\right)=\left(2 / \xi_{1}, 2 /\left(2-\xi_{1}\right)\right)$ and $\left(p_{2}, q_{2}\right)=\left(2 / \xi_{2}, 2 /\left(2-\xi_{2}\right)\right)$, we obtain

$$
\begin{aligned}
\left|U_{n, m}(f ; x, y)-f(x, y)\right| \leqslant & M_{x} B_{\mathfrak{n}}\left((t-x)^{2} ; x\right)^{\xi_{1} / 2}{ }_{x} B_{\mathfrak{n}}\left(e_{0} ; x\right)^{\left(2-\xi_{1}\right) / 2} \\
& \times{ }_{y} P_{m}^{*}\left((s-y)^{2} ; y\right)^{\xi_{2} / 2}{ }_{y} P_{m}^{*}\left(e_{0} ; y\right)^{\left(2-\xi_{2}\right) / 2} .
\end{aligned}
$$

Thus we get the required result. 
Theorem 4.3. If $\mathrm{f} \in \mathrm{D}_{\mathrm{b}}\left(\mathrm{I}_{\mathrm{cd}}\right)$ and $\mathrm{D}_{\mathrm{B}} \mathrm{f} \in \mathrm{B}\left(\mathrm{I}_{\mathrm{cd}}\right)$, then for each $(x, y) \in \mathrm{I}_{\mathrm{cd}}$, we get

$$
\begin{aligned}
\left|u_{n, m}(f ; x, y)-f(x, y)\right| \leqslant & C\left\{3\left\|D_{B} f\right\|_{\infty}+2 \omega_{\text {mixed }}\left(f ; \delta_{n}, \delta_{m}\right) \sqrt{x^{2}+x} \sqrt{y^{2}+y+1}\right\} \delta_{n} \delta_{m} \\
& +\left\{\omega _ { \text { mixed } } ( f ; \delta _ { n } , \delta _ { m } ) \left(\delta_{m} \sqrt{x^{4}+x^{3}+x^{2}+x} \sqrt{y^{2}+y+1}\right.\right. \\
& \left.\left.+\delta_{n} \sqrt{y^{4}+y^{3}+y^{2}+y+1} \sqrt{x^{2}+x}\right)\right\}
\end{aligned}
$$

where $\delta_{n}=\sqrt{\frac{a_{n}}{n}}, \delta_{m}=\sqrt{\frac{\sigma(g)}{c_{m}}}, \sigma(g)$ being $\max \{\eta(g), \mu(g)\}$, and $C$ be a constant depending only on $n, m$.

Proof. By our hypothesis

$$
\Delta_{(x, y)} f(t, s)=(t-x)(s-y) D_{B} f(\alpha, \beta), \text { with } x<\alpha<t ; y<\beta<s .
$$

Clearly,

$$
D_{B} f(\alpha, \beta)=\Delta_{(x, y)} D_{B} f(\alpha, \beta)+D_{B} f(\alpha, y)+D_{B} f(x, \beta)-D_{B} f(x, y) .
$$

Since $D_{B} f \in B\left(I_{c d}\right)$, from the above equalities, we have

$$
\begin{aligned}
\left|\mathrm{L}_{n, m}\left(\Delta_{(x, y)} f(t, s) ; x, y\right)\right|= & \left|\mathrm{L}_{n, m}\left((t-x)(s-y) D_{B} f(\alpha, \beta) ; x, y\right)\right| \\
\leqslant & L_{n, m}\left(\left|t-x\|s-y\| \Delta_{(x, y)} D_{B} f(\alpha, \beta)\right| ; x, y\right) \\
& +L_{n, m}\left(|t-x \| s-y|\left(\left|D_{B} f(\alpha, y)\right|+\left|D_{B} f(x, \beta)\right|+\left|D_{B} f(x, y)\right|\right) ; x, y\right) \\
\leqslant & L_{n, m}\left(|t-x \| s-y| \omega_{\text {mixed }}\left(D_{B} f ;|\alpha-x|,|\beta-y|\right) ; x, y\right) \\
& +3\left\|D_{B} f\right\|_{\infty} L_{n, m}(|t-x \| s-y| ; x, y) .
\end{aligned}
$$

By the properties of mixed modulus of smoothness $\omega_{\text {mixed }}$, we can write

$$
\begin{aligned}
\omega_{\text {mixed }}\left(D_{B} f ;|\alpha-x|,|\beta-y|\right) & \leqslant \omega_{\text {mixed }}\left(D_{B} f ;|t-x|,|s-y|\right) \\
& \leqslant\left(1+\delta_{n}^{-1}|t-x|\right)\left(1+\delta_{m}^{-1}|s-y|\right) \omega_{\text {mixed }}\left(D_{B} f ; \delta_{n}, \delta_{m}\right) .
\end{aligned}
$$

Combining (4.3), (4.4), and using the Cauchy-Schwarz inequality we find

$$
\begin{aligned}
\left|\mathrm{U}_{n, m}(f ; x, y)-f(x, y)\right|= & \left|\mathrm{L}_{n, m} \Delta_{(x, y)} f(t, s) ; x, y\right| \\
\leqslant & 3\left\|\mathrm{D}_{B} f\right\|_{\infty} \sqrt{\mathrm{L}_{n, m}\left((t-x)^{2}(s-y)^{2} ; x, y\right)}+\left(\mathrm{L}_{n, m}(|t-x \| s-y| ; x, y)\right. \\
& +\delta_{n}^{-1} \mathrm{~L}_{n, m}\left((t-x)^{2}|s-y| ; x, y\right)+\delta_{m}^{-1} \mathrm{~L}_{n, m}\left(|t-x|(s-y)^{2} ; x, y\right) \\
& \left.+\delta_{n}^{-1} \delta_{m}^{-1} \mathrm{~L}_{n, m}\left((t-x)^{2}(s-y)^{2} ; x, y\right)\right) \omega_{\text {mixed }}\left(D_{B} f ; \delta_{n}, \delta_{m}\right) \\
\leqslant & 3\left\|D_{B} f\right\|_{\infty} \sqrt{L_{n, m}\left((t-x)^{2}(s-y)^{2} ; x, y\right)}+\left(\sqrt{L_{n, m}\left((t-x)^{2}(s-y)^{2} ; x, y\right)}\right. \\
& +\delta_{n}^{-1} \sqrt{L_{n, m}\left((t-x)^{4}(s-y)^{2} ; x, y\right)}+\delta_{m}^{-1} \sqrt{L_{n, m}\left((t-x)^{2}(s-y)^{4} ; x, y\right)} \\
& \left.+\delta_{n}^{-1} \delta_{m}^{-1} L_{n, m}\left((t-x)^{2}(s-y)^{2} ; x, y\right)\right) \omega_{\text {mixed }}\left(D_{B} f ; \delta_{n}, \delta_{m}\right) .
\end{aligned}
$$

Since, for $(x, y),(t, s) \in I_{c d}$ and $i, j \in\{1,2\}$, we have

$$
T_{n, m}\left((t-x)^{2 i}(s-y)^{2 j} ; x, y\right)={ }_{x} B_{n}\left((t-x)^{2 i} ; x\right){ }_{y} P_{m}^{*}\left((s-y)^{2 j} ; y\right),
$$


and from Lemma 2.3,

$$
\begin{aligned}
{ }_{x} B_{n}\left((t-x)^{2} ; x\right) & =O\left(\frac{a_{n}}{n}\right)\left(x^{2}+x+1\right), \\
x & B_{n}\left((t-x)^{4} ; x\right)=O\left(\frac{a_{n}}{n}\right)\left(x^{4}+x^{3}+x^{2}+x\right), \\
y & P_{m}^{*}\left((s-y)^{2} ; y\right) \leqslant \frac{\eta(g)}{c_{m}}\left(y^{2}+y+1\right) \\
y & P_{m}^{*}\left((s-y)^{4} ; y\right) \leqslant \frac{\mu(g)}{c_{m}}\left(y^{4}+y^{3}+y^{2}+y+1\right) .
\end{aligned}
$$

Combining (4.5) and (4.6) we get the required result.

\section{Acknowledgment}

The second author is thankful to "The Ministry of Human Resource and Development", India for the financial support to carry out the above work. The third author of this paper is also supported by the Research Fund of Hasan Kalyoncu University in 2017.

\section{References}

[1] P. N. Agrawal, N. İspir, Degree of approximation for bivariate Chlodowsky-Szasz-Charlier type operators, Results Math., 69 (2016), 369-385. 1, 4

[2] Ç. Atakut, İ. Büyükyazıcı, Stancu type generalization of the Favard-Szász operators, Appl. Math. Lett., 23 (2010), 1479-1482. 1

[3] C. Badea, I. Badea, H. H. Gonska, A test function theorem and approximation by pseudopolynomials, Bull. Austral. Math. Soc., 34 (1986), 53-64. 4

[4] C. Badea, I. Badea, H. H. Gonska, Notes on the degree of approximation of B-continuous and B-differentiable functions, J. Approx. Theory Appl., 4 (1988), 95-108. 4

[5] K. Bögel, Mehrdimensionale Differentiation von Funktionen mehrerer reeller Veränderlichen, (German) J. Reine Angew. Math., 170 (1934), 197-217. 4

[6] K. Bögel, Über mehrdimensionale Differentiation, Integration und beschränkte Variation, (German) J. Reine Angew. Math., 173 (1935), 5-30 . 4

[7] K. Bögel, Über die mehrdimensionale Differentiation, (German) Jber. Deutsch. Math.-Verein., 65 (1962), 45-71. 4

[8] E. Dobrescu, I. Matei, The approximation by Bernten type polynomials of bidimensionally continuous functions, (Romanian) An. Univ. Timişoara Ser. Şti. Mat.-Fiz., 4 (1966), 85-90. 4

[9] A. D. Gadjiev, H. Hacsalihoglu, Convergence of the sequences of linear positive operators, Ankara University, (1995). 3.6, 3.7, 3.8

[10] A. D. Gadžiev, Positive linear operators in weighted spaces of functions of several variables, (Russian) Izv. Akad. Nauk Azerbaĭdzhan. SSR Ser. Fiz.-Tekhn. Mat. Nauk, 1 (1980), 32-37. 3.6, 3.7, 3.8

[11] N. İspir, Ç. Atakut, Approximation by modified Szasz-Mirakjan operators on weighted spaces, Proc. Indian Acad. Sci. Math. Sci., 112 (2002), 571-578. 1, 3, 3

[12] N. İspir, İ. Büyükyazıcı, Quantitative estimates for a certain bivariate Chlodowsky-Szasz-Kantorovich type operators, Math. Commun., 21 (2016), 31-44. 3

[13] A. Jakimovski, D. Leviatan, Generalized Szász operators for the approximation in the infinite interval, Mathematica (Cluj), 34 (1969), 97-103. 1

[14] M. A. Marchaud, Sur les dérivées et sur les différences des fonctions de variables réelles, (French) NUMDAM, [place of publication not identified], (1927), 90 pages . 4

[15] M. Sidharth, N. İspir, P. N. Agrawal, GBS operators of Bernstein-Schurer-Kantorovich type based on q-integers, Appl. Math. Comput., 269 (2015), 558-568. 1, 4

[16] M. Sidharth, N. İspir, P. N. Agrawal, Approximation of B-continuous and B-differentiable functions by GBS operators of q-Bernstein-Schurer-Stancu type, Turkish J. Math., 40 (2016), 1298-1315. 4

[17] S. Varma, F. Tadelen, Szasz type operators involving Charlier polynomials, Math. Comput. Modelling, 56 (2012), 118122. 1

[18] Z. Walczak, On approximation by modified Szasz-Mirakyan operators, Glas. Mat. Ser. III, 37 (2002), 303-319. 1 